\title{
Recursos Terapêuticos Para a Fibromialgia: Uma Revisão Sistemática
}

\author{
Géssika Araújo de Melo ${ }^{1}$, Gilvane de Lima Araújo², \\ Aline Miranda de Vasconcelos ${ }^{3}$, Nelson Torro ${ }^{4}$
}

\begin{abstract}
RESUMO
A Fibromialgia (FM) é caracterizada como uma síndrome altamente prevalente e incapacitante que afeta, principalmente, mulheres na faixa etária entre 20 e 55 anos. Atualmente não há cura para FM, portanto seu tratamento visa a reduzir os sintomas, principalmente de dor, e melhorar a qualidade de vida e funcionalidade do indivíduo. Os tratamentos farmacológicos não têm sido eficazes nessa população, portanto, emerge a necessidade de terapêuticas complementares na FM. Assim, o presente estudo objetivou analisar o estado da arte acerca dos recursos terapêuticos não farmacológicos utilizados para o quadro clínico da FM. Trata-se de uma revisão sistemática da literatura, na qual se utilizaram as bases de dados da PubMed, Psycinfo, ScienceDirect, Lilacs e Scielo. Após aplicação dos critérios de elegibilidade, permaneceram 18 artigos. 0 interesse em estudos acerca dos recursos terapêuticos utilizados na FM tem sido crescente, concentrando-se nos dois últimos anos. As possibilidades terapêuticas mais estudadas nos últimos cinco anos foram os exercícios resistidos e aeróbicos, a hidroterapia, a cinesioterapia e a neuromodulação. Ressalta-se que as características individuais devem ser levadas em consideração para escolha do tratamento pela heterogeneidade dos sintomas. Por fim, sugere-se que o acompanhamento destes indivíduos seja multiprofissional em razão de o quadro clínico da FM ser multifatorial.
\end{abstract}

Palavras-chave: Fibromialgia. Fisioterapia. Dor crônica.

\section{THERAPEUTIC RESOURCES FOR FIBROMYALGIA: A SYSTEMATIC REVIEW}

\begin{abstract}
Fibromyalgia (FM) is characterized as a highly prevalent and disabling syndrome that mainly affects women aged 20 to 55 years. Currently there is no cure for FM, so its treatment aims to reduce symptoms, especially pain, and improve the quality of life and functionality of the individual. Pharmacological treatments have not been effective in this population, therefore, the need for complementary therapies in FM emerges. Thus, the present study aimed to analyze the state of the art about the non-pharmacological therapeutic resources used for the clinical picture of FM. This is a systematic literature review using PubMed, Psycinfo, ScienceDirect, LILACS and SciELO databases. After applying the eligibility criteria, 18 articles remained. Interest in studies on the therapeutic resources used in FM has been growing, focusing on the last two years. The most studied therapeutic possibilities in the last five years were resistance and aerobic exercises, hydrotherapy, kinesiotherapy and neuromodulation. It is noteworthy that individual characteristics should be taken into consideration when choosing treatment, due to the heterogeneity of symptoms that may be present in each patient. Finally, it is suggested that the follow-up of these individuals be multiprofessional because the clinical picture of FM is multifactorial.
\end{abstract}

Keywords: Fibromyalgia. Physiotherapy. Chronic pain.

RECEBIDO EM: 20/11/2019

MODIFICAÇÕES SOLICITADAS EM: 20/3/2020

ACEITO EM: 18/4/2020

\footnotetext{
Fisioterapeuta, doutoranda em Neurociência Cognitiva e Comportamento pela Universidade Federal da Paraíba. Endereço http://lattes.cnpq. br/9921954606115118. https://orcid.org/0000-0003-2938-7280. gessika.fisio@gmail.com

2 Discente de Fisioterapia do UNIESP - Centro Universitário. http://lattes.cnpq.br/5176552861098780. https://orcid.org/0003-1625-3435. gilvanearaujo18@ gmail.com

${ }_{3}$ Fisioterapeuta, docente do curso de Fisioterapia da Faculdade Internacional da Paraíba. http://lattes.cnpq.br/5701396456493228. https://orcid.org/00000002-8722-8491. alinemvasconcelos@hotmail.com

${ }^{4}$ Psicólogo, professor doutor do Departamento de Psicologia da Universidade Federal da Paraíba. http://lattes.cnpq.br/8037098495288980. http://orcid. org/0000-0003-3678-5762 nelsontorro@gmail.com
} 


\section{INTRODUÇÃO}

A Organização Mundial da Saúde (OMS), sob o código M79.7 do CID-10, define fibromialgia (FM) como uma condição de dor generalizada, associada à fadiga, alterações no sono e distúrbios cognitivos (BOERMA et al., 2016; GARCÍA; NICOLÁS; HERNÁNDEZ, 2016). Caracterizada como uma síndrome altamente prevalente e incapacitante, afeta principalmente mulheres com faixa etária entre 20 e 55 anos (HEIDARI; AFSHARI; MOOSAZADEH, 2017).

A patogênese da FM é complexa e ainda desconhecida, porém estudos recentes identificaram possíveis fatores envolvidos na sua fisiopatologia, como: alteração no gene neurotransmissor modulador da sensibilidade à dor; fatores ambientais, por exemplo, infecções; e traumas físicos e psicológicos, que coletivamente, promovem uma alteração geral nos sistemas reguladores de estresse e dor, refletindo negativamente no humor, memória, sono e desempenho cognitivo (GALVANI et al., 2019; TIRELLI et al., 2019).

Atualmente não há cura para FM, portanto seu tratamento visa reduzir a sintomatologia e melhorar a qualidade de vida do indivíduo através de tratamento farmacológico e terapias alternativas, todavia pesquisas apontam relevância clínica questionável sobre o uso de medicamentos e ressaltam os exercícios terapêuticos como uma abordagem de tratamento mais segura e com menos efeitos colaterais (AMBROSE; GOLIGHTLY, 2015; GARCÍA; NICOLÁS; HERNÁNDEZ, 2016).

Em razão da prevalência desta patologia e visando a auxiliar nas tomadas de decisão clínica, a elaboração desta pesquisa busca apresentar o que de mais recente e relevante vem sendo utilizado e têm demonstrado melhores resultados para o tratamento da FM. Nesse contexto, espera-se que as terapias não farmacológicas apresentem melhores resultados quanto à melhora da funcionalidade, sono, dor, fadiga, entre outros aspectos, do que a terapia exclusiva medicamentosa (ATZENI et al., 2019).

Sendo assim, o presente estudo objetivou analisar o estado da arte acerca dos recursos terapêuticos não farmacológicos utilizados para o quadro clínico da FM.

\section{MATERIAIS E MÉTODO}

O presente estudo tratou-se de uma revisão sistemática da literatura sobre os aspectos gerais da FM e os recursos terapêuticos utilizados no seu tratamento. Assim, realizou-se uma pesquisa nas bases de da- dos da PubMed, Psycinfo, ScienceDirect, Lilacs e Scielo com as seguintes palavras-chave: "Fibromyalgia" AND "Chronic Pain" AND "Physiotherapy" e "Fibromialgia" AND "Dolor Crónico" AND "Fisioterapia", nos meses de outubro e novembro de 2019.

Os critérios utilizados para inclusão dos artigos na pesquisa foram: estudos relevantes e relacionados ao tema; publicações em inglês, português ou espanhol; estudos com abordagem quantitativa e publicados entre 2015 e 2019. Foram excluídos textos que abordavam a FM como comorbidade e não como doença de base, artigos de protocolos para ensaios clínicos e revisões da literatura, além de estudos com modelos animais e artigos repetidos nas bases de dados. Seguiram-se os critérios do Prisma (principais itens de relatórios de revisão sistemática e meta-análise) para revisão sistemática da literatura.

A busca nas bases de dados foi realizada por dois avaliadores de forma independente, que fizeram a primeira filtragem dos artigos com base na análise do título e do resumo. Sequencialmente, um terceiro avaliador analisou os textos selecionados e verificou a duplicidade dos mesmos e, por fim, foram aplicados os critérios de exclusão.

Após a seleção final realizou-se a análise temática dos artigos na íntegra. Dessa maneira, os 18 artigos selecionados foram analisados por 3 avaliadores independentes, que preencheram uma ficha de leitura e resumo dos textos, com o intuito de selecionar as informações mais relevantes à revisão. Para esta etapa, consideraram-se as categorias definidas previamente: Autores e ano de publicação do estudo, objetivo da pesquisa, Recursos gerais utilizados para o tratamento da FM e Recursos e protocolos fisioterapêuticos empregados para FM. Com base nos achados da literatura a partir dos descritores pré-selecionados, ampliou-se a abordagem acerca dos recursos e técnicas, visando a contemplar a diversidade de tratamentos utilizados na multifatoriedade da doença.

\section{RESULTADOS}

Após a busca com os descritores predeterminados, foram encontrados 370 artigos a partir do somatório das bases de dados PubMed, SienceDirect, Lilacs e Scielo. Não foram encontrados artigos na Psycinfo. Após aplicação dos critérios de elegibilidade, permaneceram 19 artigos. O fluxo da informação referente às fases da revisão sistemática está exposto a seguir (Fluxograma 1). 
Fluxograma 1 - Informação das fases da revisão sistemática, consoante normas do Prisma

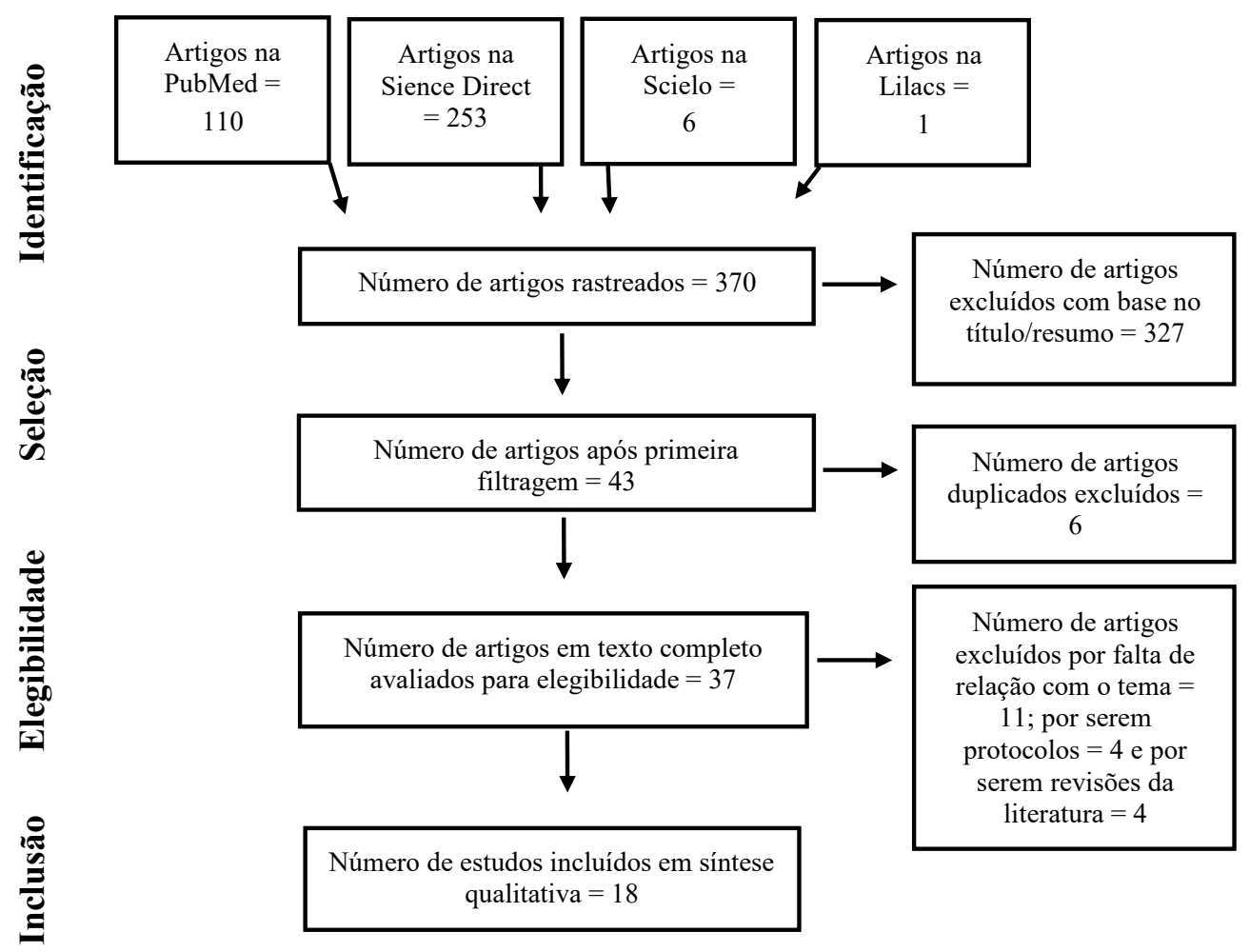

Fonte: Dados da pesquisa, 2019.

A maioria dos estudos $(n=10)$ foi publicada nos dois últimos anos, refletindo o crescente interesse pela temática. Destes, 1 correspondeu a estudo de série de casos, 1 a estudo piloto e 16 a ensaios clínicos. Os ensaios clínicos representaram a maioria, seguindo a perspectiva de comparação entre técnicas a fim de comprovar eficácia terapêutica a partir de estudos amplos, controlados e randomizados.
Quanto às principais temáticas abordadas nesses estudos, destacam-se os exercícios resistidos e aeróbicos, a hidroterapia e a termoeletrofoterapia, além de neuromodulação. As informações gerais referentes aos 18 artigos encontram-se sumarizadas na Tabela 1.

Tabela 1 - Informações gerais sobre os artigos da revisão sistemática

Autores/Ano Objetivo Protocolo utilizado Desfecho(s)

$\begin{array}{ccc}\text { Salm et al. } & \text { Avaliar o efeito da associação } & \text { Durante seis semanas ambos os } \\ 2019 & \text { dos exercícios aquáticos (EA) } & \text { grupos realizaram um programa } \\ & \text { com Emissão a distância de } & \text { de EA, } 3 \text { vezes na semana em } \\ \text { Infravermelho na cerâmica (cFIRs). } & \text { uma piscina aquecida. }\end{array}$

Castro Sánchez

et al.

2019
Comparar o agulhamento seco versus liberação miofascial nos músculos cervicais, qualidade de vida, fadiga, ansiedade e depressão na FM.
Dor, rigidez, ansiedade, depressão melhoraram após tratamento EA + cFIR. Ambos os grupos mostraram redução significativa dos níveis de IL-6 na análise intragrupo.

Quatro sessões (uma vez por semana).

O grupo de liberação miofascial melhorou apenas a intensidade da dor. O grupo de agulhamento melhorou ansiedade, depressão, dor e fadiga. 
Ozen et al. Comparar os efeitos do tratamento 2019 com as modalidades convencionais de fisioterapia com os da acupuntura na dor, na função diária e na qualidade de vida em pacientes com FM.

Uysal et al. Investigar o efeito do tratamento 2019 com MET aplicado aos músculos respiratórios acessórios cervicais na força e resistência muscular respiratória na FM.

Maciel et al. Investigar os efeitos da laserterapia 2018 de baixo nível combinada a um programa de exercícios funcionais no tratamento da FM.

Ernberg et al. Investigar o efeito de 15 semanas 2018 de exercício resistido progressivo ou relaxamento sobre os níveis de citocinas em FM e comparar com saudáveis.

Hedman- Investigar a relação custo-benefício Lagerlöf et al. da Terapia de exposição fornecida 2018. pela internet (iExp) para FM.

De Almeida Silva et al. 2018

Ahmed et al.
2018 subjacentes da estimulação do
campo nervoso occipital (ONS)
usando a Tomografia por Emissão
de Pósitrons (PET) $\mathrm{H}_{2}{ }^{15} \mathrm{O}$.

Yoo et al. 2018

Avila et al. 2017
Fisioterapia: 15 sessões (cinco sessões consecutivas por semana com duração de $1 \mathrm{~h}$ ). Acupuntura: 10 sessões de 20 min, 1 vez a cada dois dias, por três semanas.

Três dias por semana, por três semanas. A MET foi utilizada após 20 minutos de aplicação de calor superficial na região cervical.

Exercícios associados à fototerapia placebo ou ativa. Sessões de 40-60 minutos, três vezes por semana, por oito semanas.

Exercício progressivo de resistência por 10 minutos e treinamento de força de 50 minutos. O relaxamento foi o autógeno. Ambos realizados 2 vezes por semana, durante 15 semanas.

10 semanas de material e tratamento on-line com terapeutas: psicoeducação, exposição a estímulos relacionados à dor $\mathrm{e}$ treinamento em atenção plena.

Tratamento 2 vezes na semana, por 12 semanas. 0 grupo 1 realizou 2 sessões de relaxamento por 40 minutos. 0 grupo 2 realizou treinamento de resistência por 40 minutos.

Cirurgia para implante do eletrodo seguida por duas limiar subsensorial. semanas de estimulação do

Houve maior redução na intensidade da dor no grupo de fisioterapia. Em ambos os grupos houve melhora no resultado do SFMPQ3 e no FIQ.

A força e resistência muscular respiratória e a flexibilidade cervical aumentaram. A gravidade da dor, a fadiga e a incapacidade foram reduzidas.

Houve redução da dor e melhora no desempenho funcional e muscular, depressão e qualidade de vida nos dois grupos.

Houve fraca correlação entre os níveis de citocinas e as variáveis clínicas.

O protocolo não mostrou efeito anti-inflamatório sobre sintomas de FM nem nas variáveis clínicas e funcionais.

A iExp apresenta-se como um tratamento altamente rentável e traz bons resultados na FM quando comparada com nenhum tratamento.

O grupo 2 apresentou diminuição estatisticamente significativa para dor durante as avaliações e aumento da força. 0 grupo 1 apresentou redução da dor, mas não houve diferença na força muscular.

Houve melhora na dor, nas queixas de dor e na qualidade de vida e aumento na atividade no córtex cingulado anterior, que se estende para o córtex pré-frontal medial ventral para teta, alfa1, alfa2, beta1 e beta2.

Oito sessões de ETCC 2 vezes por semana. Grupo: 1) ETCC occipital simulada; 2) ETCC no nervo occipital por 20 minutos; 3) ETCC no DLPFC bilateral e antes da estimulação occipital (pré-frontal adicionado).

A hidroterapia consistiu em duas sessões semanais com 45 minutos, por 16 semanas.
A adição de ETCC bifrontal não melhorou o efeito da ETCC ativa em nenhuma das medidas de resultados clínicos testadas. A diferença entre os dois protocolos foi insignificante.

Houve melhora na dor e qualidade de vida, mas a cinemática escapular não mudou após o tratamento. 
Bjersing et al. 2017

Van Den Houte et al. 2017

Espí-López et
al.
2016

Paolucci et al. Determinar a eficácia de um 2016

Dupuis 2015

$$
\begin{gathered}
\text { campo magnético de frequência } \\
\text { extremamente baixa (ELF-MF) } \\
\text { na diminuição da dor crônica em } \\
\text { pacientes com FM. }
\end{gathered}
$$

terapêuticos do exercício aeróbico de baixo impacto sozinho ou em combinação com musicoterapia na FM.

Avaliar e entender os efeitos da fasciaterapia na FM.
Plazier et al. Determinar a segurança e eficácia 2015 da estimulação do nervo C2 no tratamento da FM.

Vaillant et al. Avaliar os efeitos de um programa 2015
Exercícios resistidos progressivos supervisionados, duas vezes por semana, durante 15 semanas.

Programa multidisciplinar em grupo por 12 semanas, com abordagem de fisioterapia, psicoeduação, psicomotricidade e terapia ocupacional.

O período de intervenção durou oito semanas. $O$ grupo 1 realizou exercício aeróbico e o grupo 2 fez exercício aeróbico com musicoterapia.

Dez sessões de exposição ao ELFMF e depois exposições placebos de forma contrabalanceada.

Tratamento de fasciaterapia por mais de nove meses.

Duas semanas de diferentes doses de estimulação, de 0,1 $\mathrm{mA}$, sublimiar e supralimiar em ordem aleatória, totalizando seis semanas.

Doze semanas, com três sessões por semana, 3 horas por sessão.
IGF-1 livre, IGFBP3 e leptina diminuíram em mulheres magras, mas não nas com excesso de peso e obesas. Além da melhora da dor, fadiga geral e melhora da força.

Incapacidade dolorosa, interferência funcional e gravidade da dor, os pacientes tiveram melhora mantida. A ansiedade e cinesiofobia também melhoraram. Depressão e enfrentamento da dor não melhoraram.

O grupo 1 melhorou depressão, qualidade de vida, desconforto geral e equilíbrio. 0 grupo 2 melhorou o desconforto geral.

\section{O tratamento reduziu a dor} e a manteve menor que no início. Houve benefício em curto prazo nos escores FIQ e HAQ.

Houve melhora da dor em três participantes. Todos os relataram que a fasciaterapia aliviou a dor durante a sessão, além da melhora no sono.

Redução geral no FIQ na dor e melhora no impacto nas atividades e qualidade da vida, mantida após seis meses.

Houve redução na intensidade da dor. Nos dois pontos testados e em todos os níveis de pressão houve diminuição da dor percebida.

Legenda: SF-MPQ3: Formulário abreviado do Questionário de Dor de McGill; FIQ: Questionário de Impacto da Fibromialgia; MET: Técnica de Energia Muscular; ETCC: Estimulação Transcraniana por Corrente Contínua; ONS: Estimulação do Campo Nervoso Occipital; HAQ: Questionário de Avaliação de Saúde.

Fonte: Dados da pesquisa, 2019.

\section{DISCUSSÃO}

A presente revisão sistemática buscou analisar os recursos terapêuticos não farmacológicos utilizados para o tratamento da FM nos últimos cinco anos. Dessa forma, sumarizou informações que podem embasar as tomadas de decisão clínica e demonstrar as possibilidades terapêuticas disponíveis, capazes de englobar a diversidade de perfis de pacientes com esta doença.

A fisioterapia convencional, por meio da utilização de laserterapia de baixo nível (LLLT) combinada a um programa de exercícios funcionais no tratamento de 22 mulheres com $\mathrm{FM}$, resultou em redução da dor e melhora no desempenho funcional e muscular, depressão e qualidade de vida (MACIEL et al., 2018). Ozen et al. (2019) compararam os efeitos do tratamento entre as modalidades de Fisioterapia com Transcutaneous electrical nerve stimulation (Tens), UItrassom e Hotpack versus acupuntura. Avaliaram dor, atividades de vida diária e qualidade de vida e perceberam que a Fisioterapia convencional e a acupuntura podem ser usadas no tratamento da FM, e que um método de tratamento não é superior ao outro.

Outras terapêuticas que vêm apresentando benefícios na FM são o agulhamento a seco e a liberação miofascial. Ao comparar as duas técnicas observou-se que a terapia com agulhamento a seco deve ser considerada uma técnica de fisioterapia dentro de uma abordagem multidisciplinar, pois reduziu a dor, dimi- 
nuiu os sintomas de ansiedade, depressão e fadiga e melhorou a qualidade de vida e o sono em curto prazo (CASTRO SÁNCHEZ et al., 2019). Em contrapartida, os mesmos autores afirmam que a liberação miofascial reduziu apenas a intensidade da dor e o impacto dos sintomas da FM após quatro sessões. Os achados supra corroboram os resultados do estudo de Dupuis (2015), que avaliou os efeitos da fasciaterapia em seis pacientes com FM que realizaram os atendimentos por mais de nove meses, resultando em redução da intensidade da dor.

De Almeida Silva et al. (2018) e Ernberg et al. (2018) buscaram investigar os efeitos das técnicas de relaxamento comparadas à prática de exercícios. No primeiro estudo, o treinamento resistido foi mais eficaz que a técnica Sophrology para melhora da força e capacidade funcional das mulheres com FM. O segundo demonstrou que em 15 semanas de tratamento os exercícios resistidos não mostraram efeito anti-inflamatório sobre os sintomas de FM nem nas variáveis clínicas e funcionais.

A prática de exercícios também foi abordada no estudo de Bjersing et al. (2017), que avaliaram fatores metabólicos em mulheres magras, com sobrepeso e obesas durante o exercício resistido e sua relação com a gravidade dos sintomas em 43 mulheres, com idades entre 25 e 64 anos. Eles perceberam que mulheres magras respondem melhor ao tratamento e que, por isso, é essencial a associação do exercício resistido com intervenções alimentares para beneficiar pacientes com FM e excesso de peso.

No tocante aos exercícios aquáticos, Salm et al. (2019) avaliaram o efeito da sua associação com a Emissão a distância de Infravermelho na cerâmica (cFIRs) sobre marcadores bioquímicos de dor e inflamação, limiares de dor e qualidade de vida em 28 muIheres com FM. Desses autores destacaram nos seus resultados que a combinação dos tratamentos beneficiou a qualidade de vida do paciente, atuando com efeito anti-inflamatório. Enquanto isso, Avila et al. (2017) utilizaram apenas a hidroterapia, com protocolo de 16 semanas, com enfoque na cinemática escapular e melhora da sensibilidade à dor. Observaram que houve melhora importante na dor e na qualidade de vida, porém a cinemática escapular não foi alterada após o tratamento.

Outra estratégia utilizada é o treinamento respiratório, com o intuito de reduzir dor e otimizar a função respiratória. Assim, Uysal et al. (2019) e Vaillant et al. (2015) investigaram os efeitos de um programa de reabilitação associado ao treino respiratório. Ambos apresentam melhorias nos sistemas musculoesqueléticos e cardiorrespiratórios, mostrando benefícios nos mecanismos de regulação da dor na FM.

Tratamentos convencionais associados às terapêuticas alternativas também vêm ganhando espaço. Nesse sentido, a prática de exercícios físicos, associados à musicoterapia, foi investigada por Espí-López et al. (2016), que demonstram que o exercício aeróbico é eficaz para melhorar a depressão e o desconforto geral na FM. A eficácia, no entanto, é maximizada quando combinada à musicoterapia.

Outro recurso que tem mostrando melhorias consideráveis no tratamento da FM é a neuromodulação. Ahmed et al. (2018) utilizaram a estimulação do campo nervoso occipital (ONS) para entender os efeitos neurais subjacentes na FM. Para tanto, avaliaram sete mulheres e observaram, após o tratamento, redução da dor óssea e articular e melhora da qualidade de vida, além de perceber que o ONS medeia o componente afetivo da dor e modula a via descendente desta. No estudo de Plazier et al. (2015) os autores estimularam o nervo C2 para verificar a segurança e a eficácia no tratamento da FM em 44 indivíduos. Obtiveram melhora da qualidade de vida, do impacto das atividades e da dor, constatando a eficácia e a segurança da estimulação. Yoo et al. (2018) combinaram dois alvos de estimulação, utilizando a estimulação transcraniana por corrente contínua em 34 indivíduos com FM, e descobriram que a ETCC foi suficiente para melhorar os sintomas da FM.

Formas alternativas de tratamento que podem auxiliar a terapia convencional são citadas nos estudos de Hedman-Lagerlöf et al. (2018) e Paolucci et al. (2016). No primeiro estudo os autores investigaram a relação custo-benefício da Terapia de exposição fornecida pela internet (iExp) para FM em 140 pessoas. Nesse cenário, observaram que a iExp é um tratamento rentável com bons resultados na FM, capaz de reduzir gastos com o tratamento clínico tradicional. No segundo estudo, foi utilizado um campo magnético de frequência extremamente baixa (ELF-MF) para diminuição da dor crônica em 37 mulheres, com idade média de 49 anos, durante 10 sessões. Os autores concluíram que o ELF-MF pode ser usado em uma abordagem multimodal para atenuar a dor na FM e melhorar a eficácia da terapia medicamentosa ou fisioterapêutica convencional.

A FM é uma doença multifatorial e, considerando que alguns estudos citados não obtiveram resultados significativos, a abordagem multidisciplinar pode ser uma resposta para melhorar a eficácia terapêutica. Nesse âmbito, Van Den Houte et al. (2017), por meio 
de um programa multidisciplinar que envolveu psiquiatras, fisioterapeutas, psicólogos, terapeutas ocupacionais e enfermeiros, examinaram os diferentes padrões de progresso clínico e a interação dinâmica entre preditores (depressão, ansiedade, enfrentamento da dor e cinesiofobia) em 153 pessoas com FM. O protocolo foi realizado durante 12 semanas e os autores observaram melhoria nos quadros de cinesiofobia e ansiedade, porém não houve resultados significativos na depressão e enfretamento da dor, enfatizando que o tratamento precisa se adaptar às necessidades individuais para melhorar a eficácia geral da intervenção.

A presente pesquisa apresentou como limitação o fato de ter inserido não apenas ensaios clínicos randomizados. Apenas três estudos, entretanto, não seguiram esses critérios e foram incluídos por suas relevâncias para a temática abordada.

\section{CONCLUSÕES}

O presente estudo sumarizou os principais achados acerca das possibilidades de tratamentos não farmacológicos que têm sido utilizados nos últimos anos, com o intuito de auxiliar profissionais da saúde nas tomadas de decisão clínica. $O$ interesse em pesquisa envolvendo recursos terapêuticos utilizados na FM tem sido crescente, concentrando-se nos dois últimos anos. A ineficiência da farmacoterapia tem instigado cientistas a se dedicarem às possibilidades terapêuticas alternativas, com o intuito de maximizar a qualidade de vida dessa população, reduzindo o sofrimento decorrente do amplo quadro clínico da FM.

As possibilidades terapêuticas mais estudadas foram os exercícios resistidos e aeróbicos, a hidroterapia, a cinesioterapia e a neuromodulação. A diversidade de técnicas empregadas para a FM, assim como a variabilidade na aplicação destas, impossibilitou uma avaliação padronizada e a sugestão de um protocolo de referência para a FM.

Ressalta-se que as características individuais devem ser levadas em consideração para a escolha do tratamento em virtude da heterogeneidade dos sintomas, que podem estar presentes em cada paciente. Por fim, sugere-se que o acompanhamento seja multiprofissional, composto por fisioterapeuta, médico, psicólogo, terapeuta ocupacional e nutricionista, em razão de o quadro clínico da FM ser multifatorial.

\section{REFERÊNCIAS}

AHMED, S. et al. The effect of occipital nerve field stimulation on the descending pain pathway in patients with fibromyalgia: a water PET and EEG imaging study. BMC Neurology, v. 18, n. 1, p. 191-200, 2018.
AMBROSE, K. R.; GOLIGHTLY, Y. M. Physical exercise as non-pharmacological treatment of chronic pain: why and when. Best practice \& Research Clinical Rheumatology, v. 29, n. 1, p. 120-130, 2015.

ATZENI, F. et al. One year in review 2019: fibromyalgia. Clinical and Experimental Rheumatology, v. 37, p. 3-10, 2019.

AVILA, M. A. et al. Effects of a 16-week hydrotherapy program on three dimensional scapular motion and pain of women with fibromyalgia: a single-arm study. Clinical Biomechanics, v. 49, p. 145-154, 2017.

BJERSING, J. L. et al. Benefits of resistance exercise in lean women with fibromyalgia: involvement of IGF-1 and leptin. BMC Musculoskeletal Disorders, v. 18, n. 1, p. 106-114, 2017.

BOERMA, T. et al. Revising the ICD: explaining the WHO approach. The Lancet, v. 388, n. 10.059, p. 2.476-2.477, 2016.

CASTRO SÁNCHEZ, A. M. et al. Improvement in clinical outcomes after dry needling versus myofascial release on pain pressure thresholds, quality of life, fatigue, pain intensity, quality of sleep, anxiety, and depression in patients with fibromyalgia syndrome. Disability and Rehabilitation, v. 41, n. 19, p. 2.235-2.246, 2019.

DE ALMEIDA SILVA, H. J. et al. Sophrology versus resistance training for treatment of women with fibromyalgia: A randomized controlled trial. Journal of Bodywork and Movement Therapies, v. 23, n. 2, p. 382-389, 2018.

DUPUIS, C. An exploratory study on the effects of DBM fasciatherapy on a population suffering from fibromyalgia. Physiotherapy, v. 101, p. 336-337, 2015.

ERNBERG, M. et al. Plasma Cytokine levels in fibromyalgia and their response to 15 weeks of progressive resistance exercise or relaxation therapy. Mediators of Inflammation, v. 2.018, p. 1-14, 2018.

ESPÍ-LÓPEZ, G. V. et al. Effect of low-impact aerobic exercise combined with music therapy on patients with fibromyalgia. A pilot study. Complementary Therapies in Medicine, v. 28, p. 1-7, 2016.

GALVANI, C. et al. Postural counseling represents a novel option in pain management of fibromyalgia patients. Journal of Pain Research, v. 12, p. 327-337, 2019.

GARCÍA, D. Á.; NICOLÁS, I.; HERNÁNDEZ. Clinical approach to fibromyalgia: synthesis of evidence-based recommendations, a systematic review. Reumatología Clínica (English Edition), v. 12, n. 2, p. 65-71, 2016.

HEIDARI, F.; AFSHARI, M.; MOOSAZADEH, M. Prevalence of fibromyalgia in general population and patients, a systematic review and meta-analysis. Rheumatology International, v. 37, n. 9, p. 1.527-1.539, 2017.

HEDMAN-LAGERLÖF, M. et al. Cost-effectiveness and cost-utility of internet-delivered exposure therapy for fibromyalgia: results from a randomized, controlled trial. The Journal of Pain, v. 20, n. 1, p. 47-59, 2018.

MACIEL, D. G. et al. Low-level laser therapy combined to functional exercise on treatment of fibromyalgia: a double-blind randomized clinical trial. Lasers in Medical Science, v. 33, n. 9, p. 1.949-1.959, 2018. 
OZEN, S. et al. A Comparison of Physical Therapy Modalities Versus Acupuncture in the Treatment of Fibromyalgia Syndrome: A Pilot Study. The journal of Alternative and Complementary Medicine, v. 25, n. 3, p. 296-304, 2019.

PAOLUCCI, T. et al. Efficacy of extremely low-frequency magnetic field in fibromyalgia pain: A pilot study. Journal of Rehabilitation Research \& Development, v. 53, n. 6, p. 1.023-1.034, 2016.

PLAZIER, M. et al. C2 nerve field stimulation for the treatment of fibromyalgia: a prospective, double-blind, randomized, controlled cross-over study. Brain Stimulation, v. 8, n. 4, p. 751-757, 2015.

SALM, D. C. et al. Aquatic exercise and Far Infrared (FIR) modulates pain and blood cytokines in fibromyalgia patients: A double-blind, randomized, placebo-controlled pilot study. Journal of Neuroimmunology, v. 337, p. 57-77, 2019. DOI: 10.1016/j.jneuroim.2019.577077.

TIRELLI, U. et al. Ozone therapy in 65 patients with fibromyalgia: an effective therapy. European Review for Medical and Pharmacological Sciences, v. 23, n. 4, p. 1.786-1.788, 2019.

UYSAL, S. C. et al. Effectiveness of the muscle energy technique on respiratory muscle strength and endurance in patients with fibromyalgia. Journal of Back and Musculoskeletal Rehabilitation, n. Preprint, p. 1-10, 2019.

VAILLANT, J. et al. Effects of a 12-week rehabilitation program on pressure pain threshold in individuals with fibromyalgia. Physiotherapy, v. 101, p. 401-403, 2015.

VAN DEN HOUTE, M. et al. Differentiating progress in a clinical group of fibromyalgia patients during and following a multicomponent treatment program. Journal of Psychosomatic Research, v. 98, p. 47-54, 2017.

YOO, H. B. et al. Adding prefrontal transcranial direct current stimulation before occipital nerve stimulation in fibromyalgia. The Clinical Journal of Pain, v. 34, n. 5, p. 421-427, 2018. 